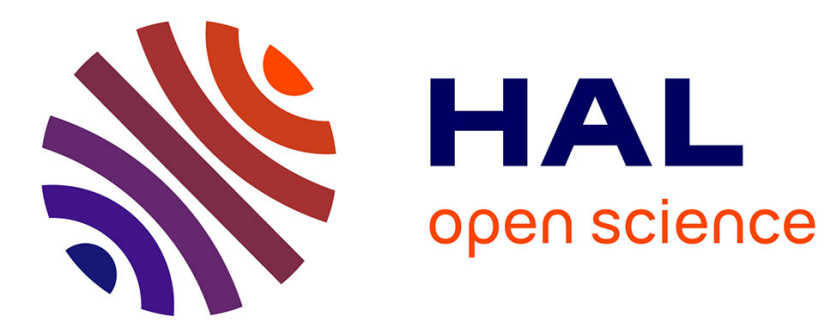

\title{
Wetting of Elastic Solids on Nanopillars
}

Maxime Ignacio, Y. Saito, P. Smereka, Olivier Pierre-Louis

\section{To cite this version:}

Maxime Ignacio, Y. Saito, P. Smereka, Olivier Pierre-Louis. Wetting of Elastic Solids on Nanopillars. Physical Review Letters, 2014, 112, pp.146102. 10.1103/PhysRevLett.112.146102 . hal-02310819

\section{HAL Id: hal-02310819 https://univ-lyon1.hal.science/hal-02310819}

Submitted on 16 Feb 2021

HAL is a multi-disciplinary open access archive for the deposit and dissemination of scientific research documents, whether they are published or not. The documents may come from teaching and research institutions in France or abroad, or from public or private research centers.
L'archive ouverte pluridisciplinaire HAL, est destinée au dépôt et à la diffusion de documents scientifiques de niveau recherche, publiés ou non, émanant des établissements d'enseignement et de recherche français ou étrangers, des laboratoires publics ou privés. 


\title{
Wetting of Elastic Solids on Nanopillars
}

\author{
M. Ignacio, ${ }^{1}$ Y. Saito, ${ }^{2}$ P. Smereka,${ }^{3}$ and O. Pierre-Louis ${ }^{1}$ \\ ${ }^{1}$ ILM, University Lyon 1, 43 Boulevard du 11 novembre 1918, 69622 Villeurbanne, France \\ ${ }^{2}$ Department of Physics, Keio University, 3-14-1 Hiyoshi, Kohoku-ku, Yokohma 223-8522, Japan \\ ${ }^{3}$ Department of Mathematics, University of Michigan, Ann Arbor, Michigan 48109, USA \\ (Received 29 November 2013; revised manuscript received 27 January 2014; published 9 April 2014)
}

\begin{abstract}
Solids and liquids are both known to exhibit Cassie-Baxter states, where a drop or a solid nanoparticle is maintained on top of pillars due to wetting forces. We point out that due to elastic strain, solid nanocrystals exhibit a behavior different from that of liquids. First, the equilibrium Cassie-Baxter state on a single pillar exhibits a spontaneous symmetry breaking due to elastic effects. The second consequence of elasticity is the existence of stable partially impaled states, resulting from a compromise between wetting forces which favor impalement and elastic strain which resists impalement. Based on kinetic Monte Carlo simulations which include elastic strain, we discuss these effects and we propose a global phase diagram for the stability of nanocrystals on nanopillars.
\end{abstract}

DOI: 10.1103/PhysRevLett.112.146102

PACS numbers: 68.55.-a, 81.10.Aj

Both liquid drops and solid nanocrystals have been experimentally observed in stable configurations on top of pillars. For liquids, this state is called the Cassie-Baxter (CB) state [1]. It is stabilized by wetting forces, and is also known as the Lotus effect. Indeed, nature uses the properties of the CB states, e.g., on plant leaves and animal skins to keep them dry and to eliminate dust via the beading and rolling of water droplets [2]. Motivated by its potential applications, notably in microfluidics, many studies have been devoted to the liquid CB state in the past decade [3]. These experimental and theoretical studies usually consider millimeter-scale drops on micron-scale pillars.

At a smaller scale, it has been pointed out already 30 years ago [4] that depositing solids on nanoscale pillars could be useful to relieve strain so as to avoid the formation of dislocations, which are known to deteriorate the quality of micro and nanotechnological devices. The existence of states where solids are stable on top of nanopillars has then been confirmed in many experimental systems in the past 15 years, such as GaAs/Si(100) nanopillars [5], GaN/Si(100) nanopillars [6], GaN/Si nanoporous substrates [7,8], Gan/3C-SiC/Si nanopillars [9], or $\mathrm{Ge} / \mathrm{Si}(001)$ nanopillars [10]. These $\mathrm{CB}$ states with solid particles, which have sometimes been referred to as "nanoheteroepitaxy" [11], indeed exhibit a lower density of dislocations. Despite their generic observation, CB states have been investigated only recently with kinetic Monte Carlo simulations and analytical models accounting for surface diffusion and anisotropy [12-14].

However, to our knowledge, the effect of heteroepitaxial elastic strain on $\mathrm{CB}$ states has not been discussed in the literature. Bending elasticity of hairlike pillars is actually suspected to lead to complex liquid CB states, involving collective period-doubling or asymmetry of the hair shapes [15]. Strain is also known to have a strong influence on solid heteroepitaxial islands, inducing shape transitions $[16,17]$ on flat substrates, and specific positioning on surface topographic features with a small aspect ratio $[18,19]$, such as mounds [20], ridges [21,22], pits [23], and trenches [24]. In this Letter we aim to show that strain changes qualitatively the behavior of nanocrystals on nanopillars, leading to novel phenomena such as asymmetric and partially impaled $\mathrm{CB}$ states that do not exist either in liquid CB states, or in islands on smooth topographic features. We focus on the case of a nanocrystal on a single pillar, which has been observed in experiments $[6,10]$, and which naturally arises in the first stages of growth on pillar forests[5].

Let us start with a short description of our KMC model, which combines a three-dimensional KMC model for surface diffusion [12], and an elastic strain solver [25-27]. Atoms are placed on a three-dimensional cubic lattice. In the following, the lattice parameter $a$ is chosen as the length unit. Three states are possible at each site: vacuum, nanocrystal atom, or substrate atom. The substrate is frozen: substrate atoms cannot move. In order to model standard surface self-diffusion on the nanoparticle [28-30], nanocrystal atoms can hop to free nearest neighbor (NN) sites when these sites exhibit at least one crystal atom among their NN or next nearest neighbors (NNN). Two technical remarks are in order. (i) These rules actually do not forbid the evaporation of dimers. In the simulations, we consider low enough temperatures, so that this evaporation is small and has negligible impact on the particle. (ii) In order to speed-up the simulations, the motion of atoms with $5 \mathrm{NN}$ was forbidden. The hopping rate of a nanoparticle atom at the surface of the nanoparticle reads $\nu=$ $\nu_{0} \exp [-(\Delta E+\Delta W) / T]$, where $\nu_{0}$ is an attempt frequency, the temperature $T$ is in units with $k_{B}=1$, and $\Delta E$ and $\Delta W$ are, respectively, the total change of bond and elastic 
energies when detaching the atom from the crystal. We implement the dynamics by means of a standard $n$-fold KMC algorithm [31,32] for the bond energies, supplemented with a rejection scheme to account for the elastic contribution.

The bond energy contribution is $\Delta E=\sum_{i} n_{i} J_{i}$ where $n_{i}$ and $J_{i}$ are, respectively, the number of neighbors, and the strength of the bond of type $i$. The types of neighbors are: $i=1$ for particle NN, $i=2$ for particle NNN, $i=s 1$ for substrate $\mathrm{NN}$, and $i=s 2$ for substrate NNN. We define two dimensionless numbers: $\zeta=J_{2} / J_{1}$, and $\chi=J_{s 1} / J_{1}$. In order to simplify the discussion, we impose $J_{s 2} / J_{2}=$ $J_{s 1} / J_{1}$. Since the surface energies depend on $\zeta$, this parameter controls the equilibrium crystal shape [12]. For $\zeta=0$, the equilibrium shape at low temperatures is a cube with (100) facets. As $\zeta$ increases (110) and (111) facets become wider [12]. The second dimensionless parameter $\chi$, called the wettability, controls the wetting properties and plays the same role as the microscopic contact angle for liquids. More precisely, in the isotropic limit, $\chi$ is related to the contact angle $\theta$ via $\chi=(1+\cos \theta) / 2$. For arbitrary anisotropy, when $\chi \rightarrow 1$, the particle spreads completely on the substrate. For $\chi \rightarrow 0$, the particle tends to its free standing equilibrium shape and can detach from the substrate without energy cost. In the following, we shall consider $0<\chi<1$, i.e., partial wetting [33]. The value of $\chi$ varies depending on the chosen system, e.g., $\chi=0.65$ for $\mathrm{Si}(100) / \mathrm{SiO}_{2}$ (SOI system) [34-36], $\chi=$ 0.35 for $\mathrm{Pt}(111) / Y$ ttria-Stabilized- $\mathrm{ZrO}_{2}$ [37], $\chi=0.31$ for $\mathrm{Pt}(111) / \mathrm{Al}_{2} \mathrm{O}_{3}$ [38], and $\chi=0.26$ for $\mathrm{Pt}(111) / \mathrm{Si}_{3} \mathrm{~N}_{4}$ [37].

Elastic strain is calculated via the relaxation of a spring network linking nearest and next nearest neighbors everywhere in the particle and in the substrate. The details of the elastic solver are presented in Ref. [27]. We work in the isotropic elasticity limit, which is obtained by using the same spring constant $K$ for NN and NNN springs. We also use the same $K$ in the substrate and in the particle. The lattice mismatch between the two solids $\epsilon=\left(a-a_{s}\right) / a_{s}$, where $a$ and $a_{s}$ are the lattice constants of the particle and the substrate, respectively, is at the origin of the strain in the particle and in the substrate. Following Ref. [27], we approximate $\Delta W$ with the sum $w$ of the elastic energies of the springs related to the atom: $\Delta W=-C w$, with $C=1.5$ [27].

Let us first consider a nanocrystal on a single nanopillar without elastic effects (i.e., no mismatch strain $\epsilon=0$, leading to $\Delta W=0$ ). We choose $\zeta=0$ so that the lowtemperature equilibrium shape of the crystal exhibits only (100) facets (we have checked that the phenomenology is similar for $\zeta=0.2$ ). The impalement depth of the crystal by the pillar is denoted as $h^{*}$, as shown in Fig. 1(a). In addition, $r_{p}=\left(x_{p}, y_{p}\right)$ denotes the two-dimensional vector relating the position of the center of the lower facet of the nanocrystal and the center of the pillar top. Lower curves in Fig. 1(b) show the surface and interface energy $F$ without
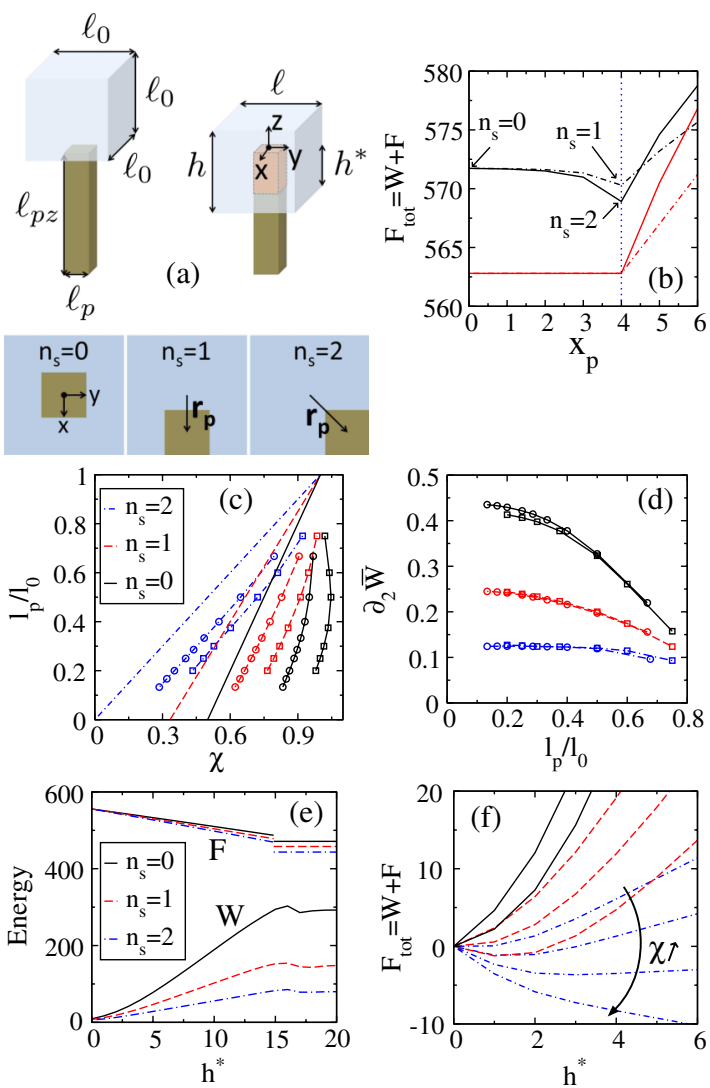

FIG. 1 (color online). Analysis of the energy of CB states. (a) Schematics of the CB configuration. The lower part of the schematics shows the relative positions of the bottom facet and the pillar top for $n_{s}=0,1,2$, and the vector $r_{p}=\left(x_{p}, y_{p}\right)$. In (b-f), $K=50$ and the nanoparticle volume is $V=14^{3}$, leading to a characteristic elastic length $\ell_{e l}=J_{1}(1+4 \zeta) /\left(5 \epsilon^{2} K a\right) \approx$ 0.82 for $\epsilon=7 \%$. (b) Variation of the total energy $F_{\text {tot }}$ in the CB state $\left(h^{*}=0\right)$ as a function of the coordinate $x_{p}$. Solid lines correspond to displacements $r_{p}=x_{p}(1,1)$, and dashed-dotted lines to $r_{p}=x_{p}(1,0)$. We have used $\ell_{p}=6$, and $\chi=0.7$ with $\epsilon=0 \%$ (lower curves, red), and $\epsilon=7 \%$ (upper curves, black). (c) Limit of stability $\chi_{c}\left(n_{s}\right)$ of the $\mathrm{CB}$ state for $n_{s}=0,1,2$ without elasticity (lines without symbols) and $\chi_{e l}$ with elasticity: Open circle for $\ell_{p}=4$, and square for $\ell_{p}=6$. (d) Data collapse of the graph (c) obtained from the derivative of the elastic energy $W$, see text. (e) Surface energy $F$ at $\chi=0.9$ and elastic energy $W$ for $\epsilon=7 \%$ as a function of the impalement depth $h^{*}$. (f) Variation of the total energy $F_{\text {tot }}$ with $\epsilon=7 \%$. We have used $\chi=$ $0.95,0.85$ for $n_{s}=0, \chi=0.95,0.85,0.75$ for $n_{s}=1$, and $\chi=$ $0.95,0.85,0.75,0.65$ for $n_{s}=2$.

elastic effects for a cubic particle of volume $V=\ell_{0}^{3}$ in the CB state with $h^{*}=0$ as a function of $x_{p}$. The energy $F$ is independent of $r_{p}$ as long as the top of the pillar is fully covered by the particle, i.e., when $x_{p} \leq\left(\ell_{0}-\ell_{p}\right) / 2$, where $\ell_{p}$ is the pillar width. However since $\chi>0, F$ increases when the top of the pillar is partially uncovered $\left(x_{p}>\left(\ell_{0}-\ell_{p}\right) / 2\right)$.

In order to analyze impalement with $h^{*} \neq 0$, three different cases shown in the lower part of Fig. 1(a) need 
to be considered. They are indexed by the number $n_{s}=$ $0,1,2$ of pillar edges in contact with a lateral facet, and have the energy

$$
\begin{aligned}
\frac{F}{\gamma_{100}}= & 2 \ell^{2}+4 h \ell-2 \chi \ell_{p}^{2} \\
& +2 \ell_{p} h^{*}\left[2-n_{s}-\left(4-n_{s}\right) \chi\right],
\end{aligned}
$$

where $h$ and $\ell$ are the height and the lateral extent of the particle, $\ell_{p}$ is the width of the pillars, and $\gamma_{100}=$ $J_{1}(1+4 \zeta) / 2 a^{2}$ is the surface energy of the (100) facet. Assuming that the impalement is slow, we may minimize $F$ with respect to $\ell$ and $h$ at fixed $h^{*}$ and fixed volume $V$, leading to $h=\ell=\left(V+h^{*} \ell_{p}^{2}\right)^{1 / 3}$. An instability of the CB state appears if $\left.\partial_{h^{*}} F\right|_{h^{*}=0}<0$. This criterion is rewritten as $\chi>\chi_{c}$, with

$$
\chi_{c}\left(n_{s}\right)=\frac{1}{4-n_{s}}\left[2-n_{s}+2 \frac{\ell_{p}}{\ell_{0}}\right],
$$

where $\ell_{0}=V^{1 / 3}$ is the lateral extent of the nanocrystal before collapse. As seen in Fig. 1(c), the impalement threshold depends on the reduced volume $\ell_{0} / \ell_{p}$, as already observed for liquid [39] and solid [12] CB states.

If the temperature is high enough, and the size of the particle small enough [40-42], the particle will diffuse laterally. Since $\chi_{c}(2) \leq \chi_{c}(1) \leq \chi_{c}(0)$, the particle is most stable in the $n_{s}=0$ configuration, but thermal fluctuations can lead to lateral diffusion to unstable positions with $n_{s}=1$ or $n_{s}=2$.

When the CB state is unstable (i.e., $\chi>\chi_{c}$ ), the energy $F$, plotted in the top part of Fig. 1(e), decreases monotonically with $h^{*}$. As a consequence, once the particle is unstable, it is impaled irreversibly. On short pillars, this process stops when the nanoparticles touches the substrate, reaching the so-called Wenzel state [43]. On long-enough pillars, the collapse proceeds up to the configuration where the top facet of the particle reaches the top of the pillar. Then, the nanoparticle experiences a flat energy landscape when moving along the pillar. As a consequence, and as already discussed in Ref. [14] for multiple pillars, the particle diffuses along the pillar if its mobility is large enough.

Let us now turn to the consequences of elasticity, which are the central aim of this Letter. Here, strain is induced by the lattice mismatch $\varepsilon$ between the two solids. The first consequence of a nonvanishing mismatch is to break the translational invariance of the elastic energy with respect to lateral drifts in the CB state (i.e., at $h^{*}=0$ ). Indeed, when the contact zone between the pillar and the particle approaches the edges and the corner of the lower facet, more mismatch strain is relaxed and the elastic energy decreases. As a consequence, the nanoparticle now drifts irreversibly toward the edges and the corners. Using our elastic solver on static configurations, we have calculated the total elastic energy $W$ in the CB state. As shown by the upper curves in Fig. 1(b), the total energy $F_{\text {tot }}=F+W$ is minimum at the corner. Pushing the nanoparticle further away to uncover a part of the pillar top surface again leads to an increase of the surface and interface energies as seen in Fig. 1(b). As a consequence, the equilibrium configuration of a stable $\mathrm{CB}$ state is the asymmetric corner configuration $\left(n_{s}=2\right)$.

Let us now analyze the limit of stability of the CB state. Since the impalement costs additional elastic energy, we intuitively expect an extension of the domain of stability of the CB state. In order to investigate this effect, we write the total energy $F_{\text {tot }}$ of the state impaled with the depth $h^{*}$ in a dimensionless form,

$\frac{F_{\mathrm{tot}}}{\gamma_{100} \ell_{p}^{2}}=\bar{F}\left(\frac{\ell}{\ell_{p}}, \frac{h^{*}}{\ell_{p}}, \chi, n_{s}\right)+\frac{\ell_{p}}{\ell_{e l}} \bar{W}\left(\frac{\ell}{\ell_{p}}, \frac{h^{*}}{\ell_{p}}, \frac{r_{p}}{\ell_{p}}\right)$,

where $\quad \ell_{e l}=\gamma_{100} /\left(\epsilon^{2} Y\right)=J_{1}(1+4 \zeta) /\left(5 \epsilon^{2} K a\right), \quad \bar{F}=$ $F /\left(\gamma_{100} \ell_{p}^{2}\right)$, and $\bar{W}=\ell_{e l} W /\left(\gamma_{100} \ell_{p}^{3}\right)$. Using Eq. (1) the stability condition $\left.\partial_{h^{*}} F_{\text {tot }}\right|_{h^{*}=0}<0$ is written as $\chi<\chi_{e l}$, with

$$
\chi_{e l}=\chi_{c}+\frac{\ell_{p}}{\ell_{e l}} \frac{\partial_{2} \bar{W}\left(\frac{\ell}{\ell_{p}}, 0, \frac{r_{p}}{\ell_{p}}\right)}{2\left(4-n_{s}\right)},
$$

where $\partial_{2}$ denotes the derivative with respect to $h^{*} / \ell_{p}$. The term $\partial_{2} \bar{W}\left(\ell / \ell_{p}, 0, r_{p} / \ell_{p}\right)$ summarizes the influence of the full elastic strain distribution on the stability criterion. We have determined this contribution numerically with our elastic solver on static configurations for three values of $r_{p} / \ell_{p}$, corresponding to the center, edge, and corner with $n_{s}=0,1,2$, respectively. The results are plotted in Fig. 1(d), and the related increase of the stability of the CB state is shown in Fig. 1(c).

The length scale $\ell_{e l}$ can vary from ängströms to hundreds of nanometers: $\ell_{e l} \approx 0.23 \mathrm{~nm}$ for $\mathrm{GaN} / \mathrm{Si}(111)$ [6], $\ell_{e l} \approx 5.8 \mathrm{~nm}$ for $\mathrm{Ge} / \mathrm{Si}(001)$ [10], $\ell_{e l} \approx 15 \mathrm{~nm}$ for $\mathrm{GaAs} / \mathrm{Si}(100)$ [5], $\ell_{e l} \approx 420 \mathrm{~nm}$ for $\mathrm{GaN} / \mathrm{Cu}(110)$ [44]. As a consequence, the ratio $\ell_{p} / \ell_{e l}$ entering in Eq. (4) can span a wide range of values depending on the system. In contrast, we see in Fig. 1(c,d) that the elastic contribution to the stability criterion changes at maximum by a factor of two when varying $\ell_{p} / \ell_{0}$. Hence, the most crucial experimental parameter to control the increase of the $\mathrm{CB}$ stability is the prefactor $\ell_{e l} / \ell_{p}$ in Eq. (4).

We shall now discuss the case where the $\mathrm{CB}$ state is unstable, i.e., $\chi>\chi_{e l}$. As seen from Eq. (1) and shown in Fig. 1(e), the energy $F$ is essentially linear in $h^{*}$ (except for the dependence of $\ell=h=\left(V+h^{*} \ell_{p}^{2}\right)^{1 / 3}$ which is slower than linear and small when $\ell_{p} \ll \ell$ ). In contrast, the dependence of $W$ on $h^{*}$ appears to be faster than linear. Indeed, when $h^{*}$ is small, the relaxation of the additional stress resulting from an increase of $h^{*}$ benefits from the 


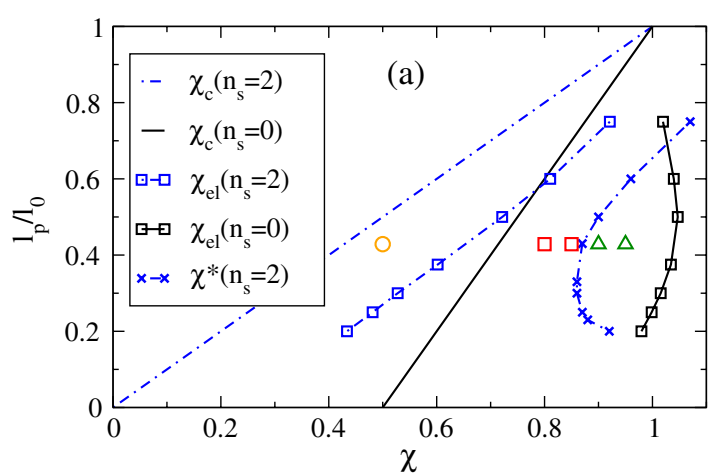

(1)
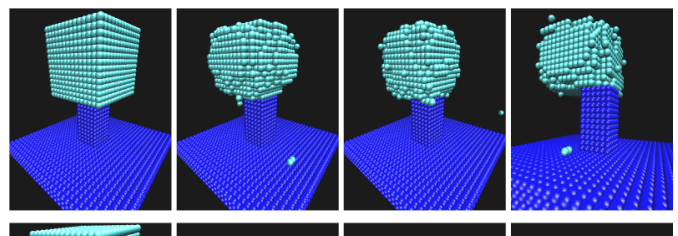

(2)
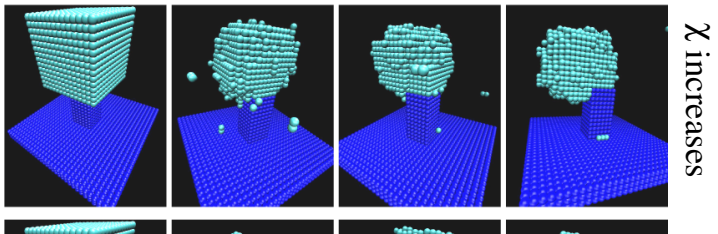

(3)
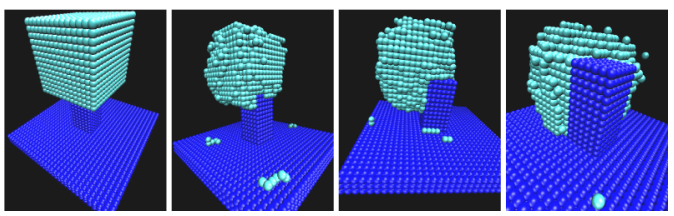

(b)

FIG. 2 (color online). Kinetic Monte Carlo simulations. (a) Phase diagram of the solid CB state with $\chi_{c}, \chi_{e l}$, and $\chi^{*}$, for $V=14^{3}, \ell_{p}=6$ and $\epsilon=7 \%$ (lines with symbols), $\epsilon=0 \%$ (lines without symbols). KMC simulation results: Open circle $\mathrm{CB}$ state, square partial impalement and triangle total impalement. (b) Snapshots of KMC simulations: (1) asymmetric $\mathrm{CB}$ at $\chi=0.5$, total simulation time $3.4 \times 10^{7} \nu_{0}^{-1}$; (2) partial impalement at $\chi=0.85$, total simulation time $6.1 \times 10^{7} \nu_{0}^{-1}$; (3) total impalement at $\chi=0.95$, total simulation time $3.0 \times 10^{7} \nu_{0}^{-1}$.

presence of the neighboring surfaces of the bottom of the nanocrystal and of the pillar. However, when $h^{*} \gg \ell_{p}$, the increase of $h^{*}$ produces additional stress far from any surface, which is less relaxed. Therefore, the increase of elastic energy $W$ as a function of $h^{*}$ is faster for large $h^{*}$, resulting in a concave profile of $W$, shown in Fig. 1(e). In the simulations, we have assumed for simplicity a collapse at fixed width $\ell=\ell_{0}$. This assumption is a priori strictly valid for $\ell \gg \ell_{p}$ only; however, in our opinion, it still catches the main qualitative behavior of the system for $\ell / \ell_{p}$ of the order of one. Combining the quasilinear behavior of $F$ with the faster-than-linear behavior of $W$, we see in Fig. 1(f) for $\chi$ slightly larger than $\chi_{e l}$, the total energy $F_{\text {tot }}=F+W$ exhibits a minimum for a special value of $h^{*}$, leading to a partially impaled state. However the partially impaled states exist only for small enough $\chi$, and for $\chi>\chi^{*}$ the minimum disappears and the impalement is complete.

Once again, the ultimate fate of the collapsing particle depends on the height of the pillars. While the collapse on short pillars leads to a Wenzel state, the collapse on high pillars leads to complete impalement as in Ref. [14].

We summarize our main results in a phase diagram describing the stability of CB states in Fig. 2(a). We have reported $\chi_{c}$ the limit of stability of the CB state without elasticity, $\chi_{e l}$ the extended limit of stability with elasticity, and $\chi^{*}$ the limit of stability of the partially impaled state. Full KMC simulations represented by the symbols, are in good agreement with the predictions. Snapshots of the simulations in Fig. 2(b) indicate that we recover the predicted asymmetric CB state, the partially impaled CB state, and the collapsed Wenzel state.

In conclusion, we have presented a global analysis of the stability of elastic solids in CB states. We find a behavior which is different from that of liquid CB states. Our results not only show that elasticity extends the stability of $\mathrm{CB}$ states, but also triggers spontaneous symmetry breaking, leading to asymmetric equilibrium states. In addition, elasticity induces partially impaled states. We show in the Supplemental Material [45] that partially impaled states can also be obtained in the case of a particle on several pillars. Our study provides novel guidelines for a better control of adhesion and wetting of nanoparticles on nanopatterned substrates such as nanowire arrays, and carbon nanotube forests.

In addition, since our geometry with a particle at the top of a pillar resembles that of catalytic particles which control the growth of nanotubes and nanowires [46-48], we speculate that our results could provide insights on the possible differences between liquid-state and solid-state catalytic particles in these systems. Indeed, the spontaneous asymmetry and the partial impalement of the particle could lead to asymmetric growth or instabilities of nanowires.

The authors acknowledge support from ANR 13 BS000-402 grant LOTUS, and Y.S. acknowledges support from JSPS.

[1] A. B. D. Cassie and S. Baxter, Trans. Faraday Soc. 40, 546 (1944).

[2] A. R. Parker and C. R. Lawrence, Nature (London) 414, 33 (2001).

[3] D. Quéré, Rep. Prog. Phys. 68, 2495 (2005).

[4] S. Luryi and E. Suhir, Appl. Phys. Lett. 49, 140 (1986).

[5] D. Zubia, S. Zhang, R. Bommena, X. Sun, S. Brueck, and S. Hersee, J. Electron. Mater. 30, 812 (2001).

[6] S. D. Hersee, X. Y. Sun, X. Wang, and M. N. Fairchild, J. Appl. Phys. 97, 124308 (2005).

[7] L. Macht, P. R. Hageman, S. Haffouz, andP. K. Larsen, Appl. Phys. Lett. 87, 131904 (2005).

[8] K. Y. Zang, Y. D. Wang, S. J. Chua, L. S. Wang, and S. Tripathy, Appl. Phys. Lett. 88, 141925 (2006). 
[9] R. Kemper, M. Weinl, C. Mietze, M. Hberlen, T. Schupp, E. Tschumak, J. Lindner, K. Lischka, and D. As, J. Cryst. Growth 323, 84 (2011).

[10] J. Bauer, Y. Yamamoto, P. Zaumseil, O. Fursenko, K. Schulz, G. Kozlowski, M. A. Schubert, T. Schroeder, and B. Tillack, Microelectron. Eng. 97, 169 (2012).

[11] D. Zubia and S. D. Hersee, J. Appl. Phys. 85, 6492 (1999).

[12] O. Pierre-Louis and Y. Saito, Europhys. Lett. 86, 46004 (2009).

[13] K. Takano, Y. Saito, and O. Pierre-Louis, Phys. Rev. B 82, 075410 (2010).

[14] M. Ignacio and O. Pierre-Louis, Phys. Rev. B 86, 235410 (2012).

[15] M. L. Blow and J. M. Yeomans, Langmuir 26, 16071 (2010).

[16] F. K. LeGoues, M. C. Reuter, J. Tersoff, M. Hammar, and R. M. Tromp, Phys. Rev. Lett. 73, 300 (1994).

[17] J. Tersoff and R. M. Tromp, Phys. Rev. Lett. 70, 2782 (1993).

[18] H. Hu, H. J. Gao, and F. Liu, Phys. Rev. Lett. 101, 216102 (2008).

[19] J.-N. Aqua, I. Berbezier, L. Favre, T. Frisch, and A. Ronda, Phys. Rep. 522, 59 (2013).

[20] T. Kitajima, B. Liu, and S. R. Leone, Appl. Phys. Lett. 80, 497 (2002).

[21] T. I. Kamins and R. S. Williams, Appl. Phys. Lett. 71, 1201 (1997).

[22] B. Yang, F. Liu, and M. G. Lagally, Phys. Rev. Lett. 92, 025502 (2004).

[23] H. Hu, H. Gao, and F. Liu, Phys. Rev. Lett. 109, 106103 (2012).

[24] G. Katsaros, J. Tersoff, M. Stoffel, A. Rastelli, P. AcostaDiaz, G. S. Kar, O. G. Schmidt, and K. Kern, Phys. Rev. Lett. 101, 096103 (2008).

[25] C.-H. Lam, C.-K. Lee, and L. M. Sander, Phys. Rev. Lett. 89, 216102 (2002).

[26] G. Russo and P. Smereka, Multiscale Model. Simul. 5, 130 (2006).

[27] T. P. Schulze and P. Smereka, Commun. Comput. Phys. 10, 1089 (2011).
[28] W. W. Mullins, J. Appl. Phys. 28, 333 (1957).

[29] H.-C. Jeong and E. D. Williams, Surf. Sci. Rep. 34, 171 (1999).

[30] C. Misbah, O. Pierre-Louis, and Y. Saito, Rev. Mod. Phys. 82, 981 (2010).

[31] A. B. Bortz, M. H. Kalos, and J. L. Lebowitz, J. Comput. Phys. 17, 10 (1975).

[32] K. A. Fichthorn and W. H. Weinberg, J. Chem. Phys. 95, 1090 (1991).

[33] P. G. de Gennes, Rev. Mod. Phys. 57, 827 (1985).

[34] D. T. Danielson, D. K. Sparacin, J. Michel, and L.C. Kimerling, J. Appl. Phys. 100, 083507 (2006).

[35] E. Bussmann, F. Cheynis, F. Leroy, P. Müller, and O. PierreLouis, New J. Phys. 13, 043017 (2011).

[36] F. Cheynis, E. Bussmann, F. Leroy, T. Passanante, and P. Müller, Phys. Rev. B 84, 245439 (2011).

[37] H. Galinski, T. Ryll, P. Elser, J. L. M. Rupp, A. BieberleHütter, and L. J. Gauckler, Phys. Rev. B 82, 235415 (2010).

[38] M. McLean and E. D. Hondros, J. Mater. Sci. 6, 19 (1971).

[39] H. Kusumaatmaja, M. L. Blow, A. Dupuis, and J. M. Yeomans, Europhys. Lett. 81, 36003 (2008).

[40] P. Jensen, Rev. Mod. Phys. 71, 1695 (1999).

[41] W. W. Pai, A. K. Swan, Z. Zhang, and J. F. Wendelken, Phys. Rev. Lett. 79, 3210 (1997).

[42] F. Leroy et al. (to be published).

[43] R. N. Wenzel, Ind. Eng. Chem. 28, 988 (1936).

[44] Q. Li, J. Figiel, G. Wang, H. Xu, and G. Balakrishnan, Appl. Phys. Lett. 100, 192110 (2012).

[45] See Supplemental Material at http://link.aps.org/ supplemental/10.1103/PhysRevLett.112.146102 for an analysis of nanoparticles on several pillars. We show that due to elastic effects, the stability of Cassie-Baxter states is extended, and partially impaled states are also obtained.

[46] A. D. Gamalski, J. Tersoff, R. Sharma, C. Ducati, and S. Hofmann, Phys. Rev. Lett. 108, 255702 (2012).

[47] C.-Y. Wen, M. C. Reuter, J. Tersoff, E. A. Stach, and F. M. Ross, Nano Lett. 10, 514 (2010).

[48] S. S. Yewu, V. Wang, V. Schmidt, and U. Gosele, Nat. Nanotechnol. 1, 186 (2006). 